\title{
A CCD MODULATION DETECTOR FOR A SECOND HIPPARCOS MISSION
}

\author{
E. $\mathrm{H} \varnothing \mathrm{G}$ \\ University Observatory \\ 1350 Copenhagen K, Denmark
}

\author{
L. LINDEGREN \\ Lund Observatory \\ 22100 Lund, Sweden
}

\begin{abstract}
A CCD modulation detector for a possible Hipparcos-2 mission is described. It is at least 1000 times more light efficient than the image dissector tube system used in the current Hipparcos mission. A beam combiner telescope with $29 \mathrm{~cm}$ aperture diameter could measure all 15 million stars brighter than 14th magnitude, providing accurate multi-colour photometry in addition to the astrometric parameters. Using two telescopes with different basic angles will further improve the rigidity of the resulting system of positions and proper motions. An accuracy of 0.5 milli-arcsec up to 10 th magnitude is expected for a 2.5 year mission. The modulating grid is imaged on a CCD performing a 'cophased integration' of nine separate images of the whole field of view, each image representing a different part of the modulation period.
\end{abstract}

\section{Introduction}

The use of CCD detectors in a future astrometry satellite is extremely interesting in view of the high quantum efficiency compared with the image dissector tube (IDT) of Hipparcos, and because the light of many stars can be integrated simultaneously. Until recently the small size of available CCDs did not match the required large field and high spatial resolution of such a satellite, but the devices now becoming available offer new possibilities.

Two very different ideas for the use of a CCD deserve attention. The option presented here (Høg, unpublished report, January 1992) is to place a grid in the focus, as in Hipparcos, and to image it onto a CCD, instead of the IDT of Hipparcos. In such a modulation detector the CCD is used only for intensity measurement of the light modulated by the grid and no great resolution or positional stability of the CCD is required.

Another option is to place the CCD in the focus of the telescope and to use it as an image detector, i.e., an accurate position measuring device. This requires high resolution and positional stability of the CCD and its mounting, but if these difficulties can be overcome the potentiality is extraordinary.

A CCD modulation detector may be of interest for other applications of similar nature where multiplexing of modulated light sources is required, e.g., for ground-based long-focus astrometry. The crucial requirement in the present application is to have a CCD with $4096 \times 270$ pixels and a charge transfer efficiency (CTE) of nearly 0.999998 . The CTE of today is 0.999994 but improvement is expected in the next few years.

The performance of a hypothetical Hipparcos- 2 will be compared with that of Hipparcos (here called Hipparcos-1), assuming the same aperture of $29 \mathrm{~cm}$, but using two beamcombiner telescopes in order to improve the rigidity of the great-circle measurements.

Abbreviations: ' $\mathrm{H}-1$ ' stands for the Hipparcos mission, ' $\mathrm{H}-2$ ' for Hipparcos-2, 'mas' for milli-arcsec, 'Hz' for counts/s or electrons/s, and 'PH' refers to Perryman \& Hassan (1989). 


\section{The Detector}

\subsection{FUNCTIONAL OVERVIEW}

The CCD modulation detector designed for Hipparcos-2, proposed by $\mathrm{H} ø \mathrm{~g} \&$ Chubey (1991), is shown in Fig. 1a. The long CCD contains $4096 \times(225+45)$ pixels. The upper part of 225 pixels width samples simultaneously the modulation light curves for all stars in the field of view, one of which is shown in Fig. 1b. The lower stripe of 45 pixels width, marked $I$ and $V$ in Fig. 1a, is used for photometry.

The main field of view with several hundred stars is integrated during about one millisecond by the CCD field \#9 of Fig. 1a. All CCD images are then shifted simultaneously to the right by one image (225 pixels) before a new integration. After nine integrations the nine electronic images of the whole field are shifted back to their initial positions. This 'shift cycle' (Fig. 1b) is repeated 40 times so that a 'cophased integration' of the modulation is built up. The reading is then performed at the corner marked 'Read'. The reading shall basically give only the total number of electrons in a 'pixel spot' of $3 \times 2$ pixels $=43 \times 29$ $\operatorname{arcsec}^{2}$ centered on each programme star.

Magnitudes in the colour bands $V$ and $I$ are determined for all programme stars as required for chromaticity correction. This is achieved by means of the stripe of 45 pixels width next to the main field, where the modulating grid is replaced by two colour filters. The bands $V$ and $I$ having effective wavelengths of 550 and $800 \mathrm{~nm}$ give a colour index $V-I$ that is nearly equivalent to $B-V$, but is preferred for astrophysical reasons when using CCDs as a better measure of the stellar temperature and less affected by interstellar reddening. In principle five-colour (UBVRI) photometry could be implemented, cf. Bessell (1990).

The star mapper is required for determination of the three-axes attitude and uses a separate CCD detector with $30 \times 120$ pixels.

In the main field of Hipparcos-1 the programme stars are measured sequentially by the cathode spot of the IDT which is switched between the stars. With the CCD modulation detector all stars in the field can be integrated simultaneously, and there is practically no upper limit to the number of stars in the field.

\subsection{DESCRIPTION OF INTEGRATION AND SAMPLING}

The sampling is illustrated in more detail in Fig. 1b. It is proposed to integrate during one modulation period $T_{\mathrm{g}}$ of $7.2 \mathrm{~ms}$ duration and then use the following period to shift back the nine field images to their initial positions. Thus, a shift cycle is defined as $T_{\mathrm{sc}}=2 T_{\mathrm{g}}$. The sampling interval is $T_{\mathrm{s}}=T_{\mathrm{g}} / 9=0.80 \mathrm{~ms}$. The shift of an image takes $T 2=225 \times 1 \mu \mathrm{s}=$ $0.225 \mathrm{~ms}$. This leaves $T 1=T_{\mathrm{s}}-T 2=0.575 \mathrm{~ms}$ for useful integration of light. The nine samples of the modulation curve are sufficient, according to van Leeuwen (1991, private comm.), while seven samples would be insufficient.

It takes a time of $T_{\text {back }}=9 \times 0.225 \mathrm{~ms}=2.025 \mathrm{~ms}$ to shift the nine fields back. Before doing so a waiting time of $T_{\text {wait }}=T_{\mathrm{g}}-T_{\text {back }}=5.175 \mathrm{~ms}$ is inserted. Since none of the useful fields receive any light during the waiting time, the exact value of $T_{\text {wait }}$ can be adjusted to take into account the small unavoidable variations in scan speed, thus keeping the synchronism between light modulation and sampling.

Sometimes the shift back must be one pixel less than the forward shift because the satellite scanning at $168.75 \mathrm{arcsec} / \mathrm{s}$ will move the star images by 1 pixel per $42.7 \mathrm{~ms}$. This produces an elongation by 1 pixel of the smeared star image, cf. Fig. 1a. The CCD has an extra length of $4096-18 \times 225=46$ pixels to accommodate these shifts. 


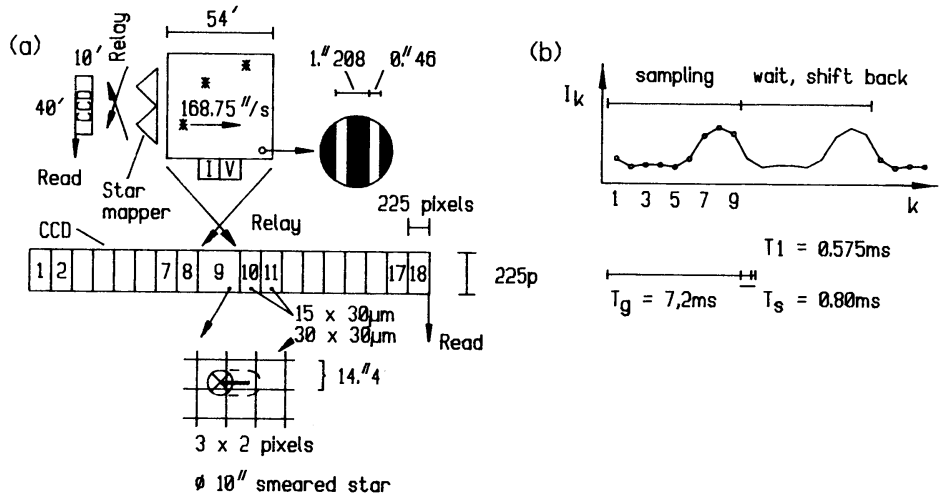

Figure 1. A CCD detector for Hipparcos-2. (a) A long CCD integrates nine points of the modulation curve for all stars in the main field. The pixel spot of $3 \times 2$ pixels contains the star image, intentionally smeared to 10 arcsec diameter by a defocussed relay optics. The image is elongated by one pixel during the integration as it moves along the fat line once per $43 \mathrm{~ms}$. (b) A shift cycle of length $2 T_{\mathrm{g}}=14.4 \mathrm{~ms}$. The nine samples are equi-spaced on one modulation period $\left(T_{\mathrm{g}}\right)$. The second period is used for shifting the charges back in preparation for the next cycle.

A shift cycle $T_{\text {sc }}$ is very nearly $=2 T_{\mathrm{g}}=14.4 \mathrm{~ms}$, but with the small variations due to $T_{\text {wait }}$. An integration cycle is $T_{\text {ic }}=N_{\mathrm{sc}} T_{\mathrm{sc}}$, where the number of shift cycles $N_{\mathrm{sc}}=40$ seems rather optimal giving $T_{\text {ic }}=576 \mathrm{~ms}$. Then the intensity of all $N_{\text {st }}$ stars are read during a time $T_{\text {rd }}=100 \mathrm{~ms}$. The number of shifts is $9 \times 225 \times 2 \times 40=162000$ pixels. After this number of shifts with a CTE of 0.999998 about 28 per cent of the electrons originally in a given pixel would be found in a neighbouring pixel, which is acceptable since it will not affect the modulation curve.

A frame is defined as a time interval $T_{\mathrm{f}}=N_{\mathrm{ic}}\left(T_{\mathrm{ic}}+T_{\mathrm{rd}}\right)$ during which the same stars are observed. With $N_{\text {ic }}=3$ we would have $T_{\mathrm{f}}=3 \times(576+100)=2028 \mathrm{~ms}$. The reading for an integration cycle gives nine numbers per star. The three sets of nine numbers in a frame can be co-added before transmission to the ground since the speed along scan is known well enough (within 0.01 per cent) to adjust the real time between integration cycles so that the three sets are in common phase.

Assuming 2 bytes per number the transmission rate for CCD data becomes $2 \times 9 \times$ $N_{\text {st }} / T_{\mathrm{f}}$ bytes/s where $N_{\text {st }}$ is the number of stars being integrated simultaneously. An input catalogue of 15 million programme stars would provide an average of 590 stars in the combined field of view per telescope. Additional information must be transmitted to the ground on the timing, star mapper observations, gyros etc. The total telemetry rate for two telescopes is then about 12 kbytes/s.

The on-board time of each sample must be known within a few $\mu$ s and must be used with the on-ground attitude in the data reduction in order to apply a phase error correction. The timing information is contained in the actual $T_{\text {wait }}$ used in each shift cycle and between the integration cycles. 


\section{Sky Background Noise}

The total photon background is expected to be nearly as in Hipparcos-1 because its IDT cathode spot has approximately the same size as the CCD spot. Before launch a total photon count background of $85 \mathrm{~Hz}$ was predicted, while the real observations have shown only one half of this, mainly because the straylight is less.

In Hipparcos- 2 with the ten times higher quantum efficiency we should therefore assume $900 \mathrm{~Hz}$ (and hope for $450 \mathrm{~Hz}$ ), mainly from sky background and straylight. The expected read noise of basically 2 electrons rms for the CCD corresponds to a background of up to $250 \mathrm{~Hz}$. The dark current of the CCD is made negligible by cooling. A total unmodulated background of $1150 \mathrm{~Hz}$ will therefore be assumed.

There is also a modulated background component due to unwanted (parasitic) stars inside the IDT spot or CCD pixel spot. As a sky average, the probability is 0.035 that a star brighter than $V=14.0$ falls inside the CCD spot in one field. The many faint stars are included in the above-mentioned unmodulated sky background, but a single bright star gives a modulated disturbance. If such a parasite in the other field of view is brighter than the programme star we assume that the observation will be rejected.

The remaining parasite disturbance has been estimated as a statistical error along scan and amounts to

$$
\sigma_{\text {parasites }}=(2.6 \mathrm{mas}) \times 10^{0.205(V-9)}
$$

per field crossing for Hipparcos-1. This assumes a spot area of $1166 \operatorname{arcsec}^{2}$, which is nearly the same as the $43 \times 29=1247 \operatorname{arcsec}^{2}$ for Hipparcos-2, so that Eq. (1) is valid also here.

\section{Photon Noise}

The photon noise of the best possible estimate of the astrometric information is given by the Cramér-Rao limit. For Poissonian noise with an intensity modulation of the form $I=I_{0}\left(1+M_{1} \cos v+M_{2} \cos 2 v\right)$, where $v$ is the modulation phase, the limiting precision is

$$
\sigma_{\text {photons }}=\frac{s}{2 \pi}\left[N \times g\left(M_{1}, M_{2}\right)\right]^{-1 / 2}
$$

where $s$ is the slit period, $N=I_{0} T$ is the total number of electrons (for integration time $T), M_{1}$ and $M_{2}$ are the modulation coefficients, and

$$
g\left(M_{1}, M_{2}\right)=\frac{1}{2 \pi} \int_{0}^{2 \pi} \frac{\left(M_{1} \sin v+2 M_{2} \sin 2 v\right)^{2}}{1+M_{1} \cos v+M_{2} \cos 2 v} d v .
$$

(These equations differ essentially from those in $\mathrm{PH}$ since their Eq. [3.5] is only correct for $M_{2} \leq 0.1$, which does not cover our case.) The background effects are taken into account by modifying $I_{0}, M_{1}, M_{2}$ as in PH. The same values $s=1.208 \operatorname{arcsec}, M_{1}=0.74$ and $M_{2}=0.26$ are used as for Hipparcos- 1 .

\section{Performance}

The light integration efficiency per reading is

$$
E_{\text {int }}=\frac{9 N_{\mathrm{sc}} T 1}{T_{\mathrm{ic}}+T_{\mathrm{rd}}}=\frac{9 N_{\mathrm{sc}} T 1}{9 N_{\mathrm{sc}}(T 1+T 2)+N_{\mathrm{sc}}\left(T_{\mathrm{wait}}+T_{\mathrm{back}}\right)+T_{\mathrm{rd}}}
$$


Table 1. Observing times, T1 and T2, for 2.5 year missions of Hipparcos-1 and -2 . The resulting photon statistical errors in columns 4 and 5 for the position components.

\begin{tabular}{rrrrr}
\hline $\begin{array}{c}V \\
\mathrm{mag}\end{array}$ & $\begin{array}{r}T 1 \\
\mathrm{sec}\end{array}$ & $\begin{array}{r}T 2 \\
\mathrm{sec}\end{array}$ & $\begin{array}{c}\mathrm{H}-1 \\
\mathrm{mas}\end{array}$ & $\begin{array}{c}\mathrm{H}-2 \\
\mathrm{mas}\end{array}$ \\
\hline 9.0 & 730 & 1880 & 0.43 & 0.08 \\
10.0 & 990 & 1880 & 0.60 & 0.14 \\
11.0 & 1330 & 1880 & 0.89 & 0.24 \\
12.0 & 1780 & 1880 & 1.41 & 0.46 \\
13.0 & 1780 & 1880 & 2.85 & 0.95 \\
14.0 & - & 1880 & - & 2.15 \\
\hline
\end{tabular}

giving 0.306 in our case. The total integration time per frame is $T_{\mathrm{f}} E_{\text {int }}=620 \mathrm{~ms}$. The largest loss of integration time is due to the shifting forth and back of the images (T2 and $\left.T_{\text {back }}\right)$ and waiting $\left(T_{\text {wait }}\right)$, while far less comes from the reading $\left(T_{\text {rd }}\right)$.

With Hipparcos- 1 the average count rate of the modulated signal for a star of $V=10 \mathrm{mag}$ is $I_{0}=707 \mathrm{~Hz}$. The ten times higher quantum efficiency of the CCD will give $I_{0}=7070 \mathrm{~Hz}$, yielding $I_{0} T_{\mathrm{f}} E_{\text {int }}=4387$ photoelectrons per frame.

The increase of light efficiency over the IDT is however not only due to the much higher detection efficiency, but also to the multiplexing advantage of observing hundreds of stars strictly simultaneously. If no loss of time is assumed for the IDT the total increase of light integration efficiency with 600 stars on the CCD is a factor $E_{\text {int }} \times 10 \times 600 \simeq 1800$ or well over 1000 . The optimum number of stars must be decided from the point of view of science, and perhaps telemetry capacity.

Overall astrometric performance values are derived as follows. The average number of field crossings for one telescope is 160 per star in a 2.5 year mission, without dead time due to Earth occultations from a geostationary orbit, and if the same scanning law as Hipparcos- 1 is assumed. A field crossing takes $19.2 \mathrm{~s}$ and gives a total time in the field of $160 \times 19.2=3072 \mathrm{~s}$. Out of this time only a part can be used for real observing, i.e., for photon integration. For Hipparcos-1 the planned IDT times per star for a 2.5 year mission are taken from Table 3.2 of $\mathrm{PH}$.

For Hipparcos-2 with two telescopes we obtain up to $2 \times 3072 \times E_{\text {int }}=1880 \mathrm{~s}$ of useful observing time per star. Table 1 gives the useful observing times and the resulting standard deviations for the position components obtained from a 2.5 year mission. These accuracy values contain photon noise, including all background and read noise. They are obtained assuming a dead time loss of 10 per cent due to Earth occultations, according to $\mathrm{PH}$ Table 3.3. The accuracy value along scan after 2.5 years is multiplied by $\sqrt{2}$ to obtain the accuracy of each position component.

The angles between all stars along scan of Hipparcos- 1 are accurately determined thanks to the large and stable basic angle between the two fields in combination with the relatively wide field of view. There is nevertheless a certain lack of 'rigidity' in the connection of stars along the great circle scan, which contributes a (magnitude independent) random error to all stars, cf. Eq. [3.10] in PH. According to Høyer et al. (1981), assuming a telescope with a single well-chosen basic angle, the rms contribution is some $25 \%$ higher than the 'typical' photon statistical error (at $V \simeq 9.0$ ), including parasites. For Hipparcos-1 this amounts to about 0.7 mas, although a precise number cannot easily be obtained for the real situation.

The non-rigidity contribution is substantially reduced by using two beam-combiner telescopes with different (incommensurable) basic angles, e.g., 58 and 74 degrees. The principle 
Table 2. Estimates of the statistical errors of the five astrometric parameters from 2.5 year missions of Hipparcos-1 and -2. The error contributions from parasitic stars, and from non-rigidity of the great circle solution are given. The resulting Total Sigma includes the photon noise and an empirical factor of 1.5. It then agrees with simulations of Hipparcos-1 in the last column.

\begin{tabular}{|c|c|c|c|c|c|c|c|}
\hline \multirow[b]{2}{*}{$\begin{array}{c}V \\
\text { mag }\end{array}$} & \multicolumn{2}{|c|}{ Parasites } & \multicolumn{2}{|c|}{ Non-Rigidity } & \multicolumn{2}{|c|}{ Total Sigma } & \multirow{2}{*}{$\begin{array}{c}\text { Sim } \\
\text { H-1 } \\
\text { mas }\end{array}$} \\
\hline & $\begin{array}{c}\mathrm{H}-1 \\
\text { mas }\end{array}$ & $\begin{array}{c}\mathrm{H}-2 \\
\text { mas }\end{array}$ & $\begin{array}{c}\mathrm{H}-1 \\
\text { mas }\end{array}$ & $\begin{array}{c}\mathrm{H}-2 \\
\text { mas }\end{array}$ & $\begin{array}{c}\mathrm{H}-1 \\
\text { mas }\end{array}$ & $\begin{array}{c}\mathrm{H}-2 \\
\text { mas }\end{array}$ & \\
\hline 9.0 & 0.3 & 0.2 & 0.7 & 0.1 & 1.3 & 0.4 & 1.5 \\
\hline 10.0 & 0.5 & 0.4 & 0.7 & 0.1 & 1.6 & 0.6 & - \\
\hline 11.0 & 0.8 & 0.6 & 0.7 & 0.1 & 2.1 & 0.9 & - \\
\hline 12.0 & 1.3 & 0.9 & 0.7 & 0.1 & 3.0 & 1.5 & 3.0 \\
\hline 13.0 & 2.0 & 1.4 & 0.7 & 0.1 & 5.3 & 2.6 & - \\
\hline 14.0 & - & 2.3 & - & 0.1 & - & 4.7 & - \\
\hline
\end{tabular}

is similar to the determination of corrections of a divided declination circle (Høg 1961), where at least two different angles are always used. A study by Makarov (1991, private comm.) indicates an improvement by up to a factor ten if two beam combiners are used, so that a contribution of the order of 0.1 mas would apply to Hipparcos- 2 .

Table 2 shows the error contributions from parasites and non-rigidity in columns 2 to 5 . A total standard error is obtained by quadratic addition of the photon noise from Table 1. However, for the standard errors in columns 6 and 7 , a further empirical factor 1.5 has been applied. This brings a fair agreement with the last column containing standard errors from simulations of Hipparcos-1, taken from Table 3.4 of $\mathrm{PH}$.

It is noted that the non-rigidity is the dominating error source for stars brighter than $V=10$ for Hipparcos-1, and even more for Hipparcos-2 if the two telescopes with different basic angles were not employed. The remaining noise from parasites, nearly equal to the photon noise, can probably only be reduced by using a smaller CCD spot.

Acknowledgements: Information on CCDs and their future was kindly provided by R.F. Nielsen and J. Janesick. Discussions at Pulkovo Observatory, and with F. van Leeuwen and M. Grenon are gratefully acknowledged. This work was supported by the Danish Space Board.

\section{References}

Bessell M.S., 1990, PASP 102, 1181.

Høg E., 1960, Astron. Nachr., 286, 65.

Høg E. \& Chubey M.S., 1991, Proposal for a Second Hipparcos, in: Proceedings of the "Etalon-91" International Symposium, Moscow 1-5 June 1991, ed. V.N. Pochucayev.

Høyer P., Poder K., Lindegren L., \& Høg E., 1981, Astron. Astrophys., 101, 228.

Makarov V., 1991, 'Periodical errors of the Hipparcos star abscissae', private comm., 6 pp.

Perryman M.A.C. \& Hassan H., 1989, The Hipparcos Mission, Volume I, The Hipparcos Satellite. ESA SP-1111. 